\title{
Depresión adolescente: factores de riesgo y apoyo social como factor protector*
}

\section{Adolescent Depression: Risk Factors and Social Support as a} Protective Factor

Recepción: 13 Julio 2015 | Aceptación: 24 Julio 2017

\author{
SANDYBell GonZÁlez LugO \\ Universidad de Sonora, México \\ ORCID: http://orcid.org/0000-0002-2918-4873 \\ Antonio Pineda Domínguez \\ Universidad de Sonora, México \\ José Concepción Gaxiola Romero \\ Universidad de Sonora, México
}

a Autor de correspondencia. Correo electrónico: sandybell.gonzalezl@gmail.com

Para citar este artículo: González Lugo, S., Pineda Domínguez, A., \& Gaxiola Romero, J. C. (2018). Depresión adolescente: factores de riesgo y apoyo social como factor protector. Universitas Psychologica, 17(3), 1-11. https://doi.org/10.11144/Javeriana.upsy1 7-3.dafr

\section{RESUMEN}

El objetivo del estudio fue evaluar, en un modelo de ecuaciones estructurales, la relación de las características negativas del vecindario y del contexto escolar, amigos de riesgo, problemas de autorregulación y sucesos de vida con la depresión en adolescentes, así como el posible papel protector del apoyo social. Previo consentimiento informado, se conformó una muestra de 113 adolescentes pertenecientes a un sector vulnerable de una ciudad del noroeste de México. Los participantes respondieron un conjunto de escalas tipo Likert validadas previamente en la región, que midieron cada una de las variables del estudio. Los resultados del modelo estructural muestran que las características negativas del contexto, los amigos y los sucesos de vida constituyen factores de riesgo para la depresión, los cuales son mitigados moderadamente por el apoyo social. Se sugiere la elaboración de programas de promoción del apoyo social en adolescentes vulnerables.

Palabras clave

depresión; adolescencia; factores de riesgo; apoyo social.

\section{ABSTRACT}

The aim of this study was to evaluate a structural equation model, the relation of negative aspects in the neighborhood, school, high-risk peers, self-regulation problems and life events with adolescent depression, as well as the protective role of social support. The subjects were 113 consenting adolescents from a vulnerable sector in a city northwest of Mexico. To measure the study's variables participants responded to a set of Likert scales which had been validated in the region. Results from the structural equation model indicate that negative aspects in the environment, friends and life events constitute risk factors for depression, which are moderately mitigated by social support. The development of programs which promote social support in vulnerable adolescents is suggested.

Keywords

depression; adolescence; risk factors; social support. 
La depresión es un periodo de al menos dos semanas en las que hay un estado de ánimo deprimido o una pérdida de interés o placer por realizar actividades, sin ser atribuible a otras condiciones médicas (American Psychiatric Association [APA], 2013). Es considerada uno de los mayores problemas de salud debido al alto grado de discapacidad que genera (Wagner, González-Forteza, Sánchez-García, García-Peña, \& Gallo, 2012). El $26 \%$ de los casos de depresión en adultos iniciaron antes de los 18 años. Estos casos de inicio temprano implican mayor probabilidad de presentar un episodio subsiguiente y mayor cantidad de episodios (Benjet, Borges, Medina, Fleiz-Bautista, \& Zambrano-Ruiz, 2004).

Los factores de riesgo relacionados con la depresión son variados e incluyen desde aspectos biológicos hasta psicológicos y sociales. Los factores de riesgo se definen como aquellos atributos o variables que incrementan la probabilidad de que personas con características similares desarrollen alguna problemática (Lucio, Rapp-Paglicci, \& Rowe, 2011). En el caso de la depresión, variables como la edad, el sexo, el divorcio, los problemas familiares y las características socioeconómicas de la región son algunos ejemplos de factores de riesgo (Díaz et al., 2006).

En población adolescente, los hallazgos científicos reportan una relación importante entre el contexto en el que viven y su salud mental (Huston \& Bentley, 2010). En este, la interacción dinámica de factores de riesgo a distintos niveles se refleja en las crecientes tasas de depresión en los adolescentes (Hankin, 2015), siendo el vecindario uno de los ambientes estudiados debido a que el lugar de residencia engloba características étnicas, económicas, de posición e inequidad social, de vivienda, de espacios públicos disponibles para la interacción social (Diez Roux \& Mair, 2010), de violencia, desorden e inseguridad (Mair, Kaplan, \& Everson-Rose, 2010) que se asocian con autorreportes de síntomas depresivos en adolescentes (Lowe et al., 2014).
Las consecuencias negativas del contexto como factor de riesgo pueden aumentar en conjunto con otras situaciones que generen estrés, como los sucesos de vida (Cutrona, Wallace, \& Wesner, 2006). Los sucesos de vida son experiencias o acontecimientos con potencial de producir estrés (Barcelata, Durán, \& Lucio, 2012), los cuales pueden suceder en diversos contextos, por ejemplo, ser víctima de violencia, de desastres naturales o sufrir la pérdida de familiares (Cutrona, Russel, \& Brown, 2005). En otras palabras, individuos que experimentan sucesos de vida estresantes en un vecindario desventajoso pueden ser más vulnerables a desarrollar depresión que individuos que viven los mismos eventos en un vecindario ventajoso (Cutrona et al., 2006).

Tradicionalmente, la depresión se ha abordado desde la teoría cognitiva, donde se ha establecido una relación entre el estilo cognitivo negativo y los síntomas depresivos, sin embargo, resultados longitudinales de investigación en adolescentes demuestran que el estilo cognitivo negativo y los síntomas de depresión no son significativos cuando no se consideran sucesos de vida estresantes (Kindt, Kleinjan, Janssens, \& Scholte, 2015).

Los sucesos de vida son fuertes predictores de la depresión y son más frecuentes en poblaciones de nivel socioeconómico bajo (Verbeek et al., 2015). La relación entre sucesos de vida tanto en el ámbito familiar, social y escolar que viven los adolescentes y los síntomas depresivos se ha reportado en investigaciones previas (Veytia, González-Arratia, Andrade, \& Oudhof, 2012). Por ejemplo, sucesos de vida estresantes relacionados con la escuela, como el bajo rendimiento, desacuerdos o problemas con los profesores, son más frecuentes en muestras clínicas con depresión que en adolescentes normales (Heredia, Lucio, \& Suárez de la Cruz, 2011).

Por otro lado, las escuelas ubicadas en sectores de población con nivel socioeconómico bajo y que reciben pocos recursos en términos de infraestructura y equipamiento, se asocian con síntomas depresivos en adolescentes a pesar del ajuste de factores individuales como edad, sexo 
y estructura familiar (Goodman, Huang, Wade, \& Kahn, 2003). Aunque los sucesos de vida en el contexto escolar son importantes, los sucesos más predictores de la depresión ocurren en la familia (Veytia et al., 2012), como maltrato físico, riñas o divorcio de los padres (Barcelata et al., 2012). A estos le siguen los problemas de tipo social, como pérdida de un amigo, apoyo social inadecuado, vivir solo, entre otros, lo cual resalta la importancia del apoyo social.

En ese sentido, las interacciones con el grupo de iguales como los amigos del adolescente también pueden resultar en un factor de riesgo (Rosenquist, Fowler, \& Christakis, 2011). El estudio de los pares y su influencia en la depresión ha sido evaluado por diversos autores. Se ha encontrado que la afiliación con pares y las cualidades positivas de los amigos se asocian negativamente con síntomas depresivos, mientras que cualidades negativas en amigos se asocian positivamente con dichos síntomas (La Greca \& Harrison, 2005). También se ha encontrado que los síntomas depresivos se dan por contagio entre pares, es decir, que los síntomas depresivos en amigos pueden predecir síntomas depresivos en un adolescente, tras controlar los síntomas depresivos iniciales de dicho adolescente (Dishion \& Tipsord, 2011). Otros estudios sugieren que la influencia de los pares en la depresión es más modesta en ciertos jóvenes. Por ejemplo, ciertos adolescentes son menos susceptibles a las influencias de los amigos debido a características como la autorregulación (Reynolds \& Crea, 2015). La autorregulación implica comportamientos que previenen la depresión y la ansiedad en adolescentes, mientras que su ausencia confiere vulnerabilidad (Ramírez \& Hernández, 2012).

Para hacer frente a estos factores de riesgo, es importante estudiar los factores protectores. En contextos de riesgo, el apoyo social se refiere al intercambio de recursos entre personas de una red, resultando en el bienestar psicosocial de los adolescentes (Auerbach, Bigda-Peyton, Eberhart, Webb, \& Ho, 2011). El apoyo social percibido puede ayudar a enfrentar los sucesos de vida estresantes y proteger de la depresión a los adolescentes, ya que se asocia con bajos niveles de estrés y depresión en jóvenes mexicanos (Raffaelli et al., 2013).

A partir de lo anterior, el objetivo del presente estudio fue evaluar el efecto de las características contextuales negativas de la escuela y el vecindario, los amigos de riesgo, problemas de autorregulación y sucesos de vida, en los indicadores de depresión de adolescentes, así como el papel protector de las redes de apoyo social como modulador de los riesgos. En la Figura 1, se muestra el modelo hipotético con las relaciones esperadas entre las variables.

\section{Figura 1}

Modelo hipotético de las relaciones entre las variables

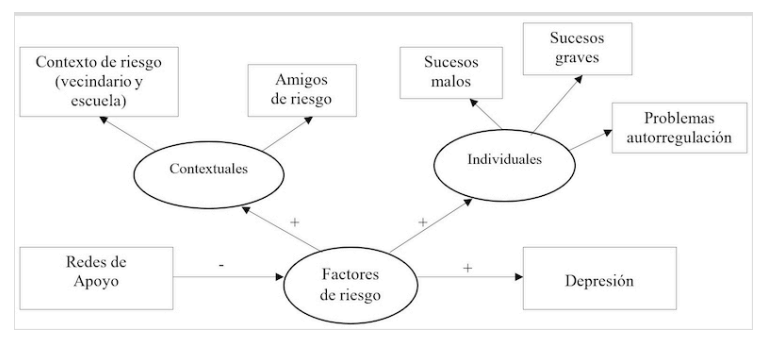

\section{Método}

Se realizó un estudio transversal-correlacional. El muestreo fue de tipo intencional, seleccionándose una escuela de nivel medio superior con bajos índices de suficiencia académica, dada la relación entre este indicador y los factores de riesgo.

\section{Participantes}

Previo consentimiento informado, se aplicó una batería de pruebas a 113 estudiantes pertenecientes a una escuela de nivel medio superior con altos índices de deserción. La edad promedio de los participantes fue de 16.33 años $(D E=1.09)$, el $59.3 \%$ eran mujeres y el $40.7 \%$ hombres. Del total de estudiantes el $54.09 \%$ provenía de familias con ingresos mensuales inferiores a 5000 pesos mexicanos. No se encontraron diferencias entre hombres y mujeres respecto a la edad $(t=1.034 ; p>$ 0.05), pero si en ingreso familiar mensual, donde 
las mujeres reportaron dos veces más frecuencia de ingresos menores a 5000 pesos mexicanos en comparación con los hombres $\left(\chi^{2}=10.89, g l=\right.$ $3 ; p<0.05)$.

\section{Instrumentos}

Inventario de Depresión de Beck-II ([BDI-II]; Beck, Steer, $\mathcal{E}$ Brown, 1996)

El inventario está conformado de 21 ítems que identifican y miden síntomas de depresión tanto en adultos como en adolescentes, a partir de los 13 años. Existe evidencia de su validez en adolescentes mexicanos, según análisis factoriales confirmatorios, así como de su confiabilidad con valores de alfa de 0.88 y 0.89 (Contreras, Hernández, \& Freyre, 2015). Ejemplos de reactivos son "no tengo ningún pensamiento de suicidio" y "me suicidaría si tuviese la oportunidad". El adolescente selecciona la opción que lo identifique durante la última semana.

Behavior Rating Inventory of Executive Functions ([BRIEF]; Gioia, Isquith, Retzlaff, Eु Espy, 2002)

La prueba evalúa problemas de autorregulación ante diversas situaciones de la vida diaria. Está conformada por 30 preguntas con siete opciones de respuesta tipo Likert que van de $0=$ nunca a $6=$ casi siempre. Algunos de sus ítems son: "las personas me dicen que yo no pienso antes de actuar" y "me incomoda cuando tengo que lidiar con cambios". Se reporta un valor de alfa de Cronbach de 0.90 en adolescentes mexicanos (Gaxiola, González, Domínguez, \& Gaxiola, 2013).

Cuestionario de Sucesos de Vida Estresores (Torres Eु Ruiz, 2013)

Adaptación de 34 ítems a partir de dos cuestionarios para adolescentes mexicanos (Lucio, León, Durán, Bravo, \& Velasco, 2001) y de Costa Rica (Villalobos-Cano, 2009). Evalúa sucesos de vida estresantes en las áreas de enfermedad o muerte, separación, sexualidad, actos delictivos y situación económica. Tiene cuatro opciones de respuesta donde el participante reporta la ocurrencia y gravedad del suceso. Ejemplos de reactivos son: "mis padres se separaron o divorciaron" y "he sido golpeado(a) o duramente castigado(a)". Se ha utilizado en investigaciones con adolescentes, correlacionándose de forma negativa con el bienestar (González \& Rivera, 2016).

\section{Escala de Características de Amigos de Riesgo} (Gaxiola, González, 83 Contreras, 2012)

Las características de las amistades consideradas de riesgo fueron evaluadas utilizando ocho ítems, en los que se indaga sobre la frecuencia de comportamientos de riesgo en los amigos del adolescente, por ejemplo, "participan en peleas o riñas" y "consumen bebidas alcohólicas". Las opciones de respuesta van de $0=$ nunca a 4 = siempre. El valor de alfa es de 0.84 según lo reportado por los autores, en una muestra de adolescentes mexicanos.

Ambiente en la Colonia (Frías, López, Ė Díaz, 2003)

Para medir las características negativas del vecindario, se utilizaron 9 reactivos de ambiente en la colonia utilizados en el estudio de Frías et al. (2003), que miden la percepción de los adolescentes acerca del vecindario, es decir su valoración subjetiva, en una escala de 0 a 10 . Algunos reactivos son: "iqué tan peligrosa es tu colonia/barrio?" y "qué tantos vagos hay en tu colonia/barrio?"; las opciones de respuesta fueron modificadas en este estudio del $0=$ nada a 4 $=$ demasiado, formato similar al de un estudio previo que reporta un valor de alfa de 0.82 en adolescentes mexicanos (Gaxiola et al., 2013). 
Ambiente Escolar (Frías, López $\mathcal{E}$ Díaz, 2003)

Esta es una escala de percepción de contexto de riesgo en las escuelas, conformada por cinco preguntas; evalúa la percepción del escolar en relación con la inseguridad y la infraestructura. La versión original maneja una forma de respuesta de 10 puntos, pero en este estudio se utilizaron cinco opciones de respuestas que van desde $0=$ nada a $4=$ demasiado. Algunas preguntas son: "iqué tan peligrosa es tu escuela?" y "iqué tan sucia está tu escuela?". Se reporta un valor de alfa de 0.78 en adolescentes con esta modalidad de respuesta (Gaxiola et al., 2013).

\section{Escala Redes de Apoyo (Villalobos-Cano, 2009)}

Torres y Ruiz (2013) adaptaron esta escala que originalmente consta de 45 reactivos, que quedó conformada por 19 ítems que evalúan el apoyo percibido de distintas fuentes por los adolescentes. Las opciones de respuesta son de cinco puntos que van de siempre a nunca; algunos de sus indicadores son: "tengo personas que me apoyan" y "cuento con algún apoyo por parte de la escuela". En un estudio previo con adolescentes mexicanos se obtuvo un valor de alfa de 0.75 (González \& Rivera, 2016).

\section{Procedimiento}

A partir de la base de datos de resultados de los exámenes de Evaluación Nacional del Logro Académico en Centros Escolares - ENLACE (Secretaría de Educación Pública, 2014), se seleccionó una escuela perteneciente a la modalidad que obtuvo menor porcentaje de resultados buenos y excelentes, debido a la relación entre la insuficiencia académica y las características negativas del entorno escolar y del vecindario (Gaxiola et al., 2012), lo cual hace probable que se encuentren entre los factores de riesgo de interés en dicha muestra.

Una vez identificado el centro escolar, se solicitó el permiso de las autoridades correspondientes, explicando los objetivos de la investigación y el proceso de la misma. Posteriormente, se seleccionaron tres grupos con la técnica de números aleatorios. Se acudió a los grupos escogidos y previo consentimiento informado, los interesados en participar respondieron el conjunto de instrumentos, así como a un apartado de datos sociodemográficos. El tiempo total de respuesta fue de aproximadamente 50 minutos.

\section{Análisis de datos}

Los datos se capturaron en el programa estadístico SPSS versión 20; se realizaron estadísticas descriptivas, análisis de confiabilidad y se conformaron índices a partir de las respuestas medias de las escalas. Se obtuvieron correlaciones entre los índices. Los índices conformados como variables latentes se exportaron al paquete estadístico multivariado EQS versión 6, donde se elaboró un modelo de ecuaciones estructurales. Para evaluar la pertinencia del modelo se utilizaron los indicadores chi cuadrado $\left(\chi^{2}\right)$ alto y no significativo, los índices Bentler-Bonett de ajuste normado (IBAN), el índice de BentlerBonnett de ajuste no normado (IBANN), así como el índice de ajuste comparativo (CFI), los cuales se espera sean cercanos a 1 (Bentler, 2006). Por último, para estimar el grado de error, se consideró el RMSEA (Raíz del Residuo Cuadrático Promedio), del cual se esperaban valores menores a 0.08 .

\section{Resultados}

En la Tabla 1 se describen los valores de alfa de Cronbach para las escalas; se observa que todas son superiores a 0.60 y por lo tanto se consideran aceptables (Nieva \& Sorra, 2003). En la Tabla 2, se describe el promedio de las respuestas para las variables de investigación. 
Tabla 1

Valores de alfa de Cronbach para las escalas del estudio

\begin{tabular}{ll}
\hline \multicolumn{1}{c}{ Variable } & $\alpha$ \\
\hline Depresión & 0.86 \\
Vecindario negativo & 0.88 \\
Amigos de riesgo & 0.81 \\
Riesgo en escuela & 0.66 \\
Sucesos de vida & 0.81 \\
Problemas de autorregulación & 0.94 \\
Redes de apoyo social & 0.78 \\
\hline \multicolumn{2}{c}{$n=113$} \\
\end{tabular}

Tabla 2

Media de las respuestas para las variables latentes de investigación

\begin{tabular}{lrr}
\hline \multicolumn{1}{c}{ Variable } & \multicolumn{1}{c}{ M } & \multicolumn{1}{c}{ DE } \\
\hline Depresión & 0.33 & 0.42 \\
Vecindario negativo & 2.54 & 0.85 \\
Amigos de riesgo & 1.71 & 0.6 \\
Riesgo en escuela & 1.9 & 0.63 \\
Sucesos de vida graves & 0.62 & 1.47 \\
Sucesos de vida malos & 1.87 & 2.2 \\
Problemas de autorregulación & 1.63 & 1.11 \\
Redes de apoyo social & 3.65 & 0.65 \\
\hline
\end{tabular}

$$
n=113
$$

La Tabla 3 muestra las relaciones significativas entre los sucesos de vida y la depresión y características de riesgo tanto a nivel vecindario, escuela y amigos, con los sucesos de vida.

Tabla 3

Correlaciones de Pearson entre las variables de investigación

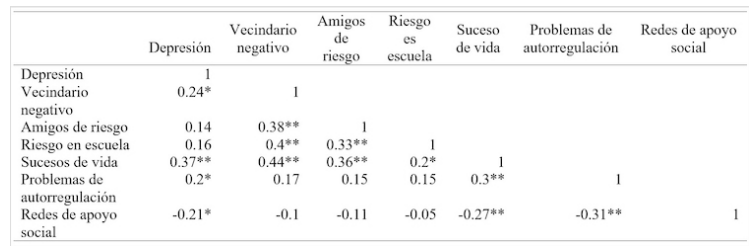

$$
\begin{aligned}
& \text { *Significativa a } p<0.05 \text { (bilateral) } \\
& \text { ** Significativa a } p<0.01 \text { (bilateral) }
\end{aligned}
$$

A partir de la literatura, se propuso un modelo teórico (Figura 1), que fue contrastado con el modelo empírico obtenido con los datos (Figura
2), donde se incluyeron todas las variables del estudio. En la Figura 2, se muestran los pesos estructurales de las relaciones entre las variables, así como los indicadores de bondad de ajuste, donde los indicadores prácticos BBNFI y BBNNFI no son cercanos a 1 (iguales o superiores a 0.95) y el valor RMSEA tampoco es inferior a 0.08 , como se sugiere para ser considerado un modelo adecuado (Ruiz, Pardo, \& San Martín, 2010).

Figura 2

Modelo con las relaciones entre todas las variables de investigación

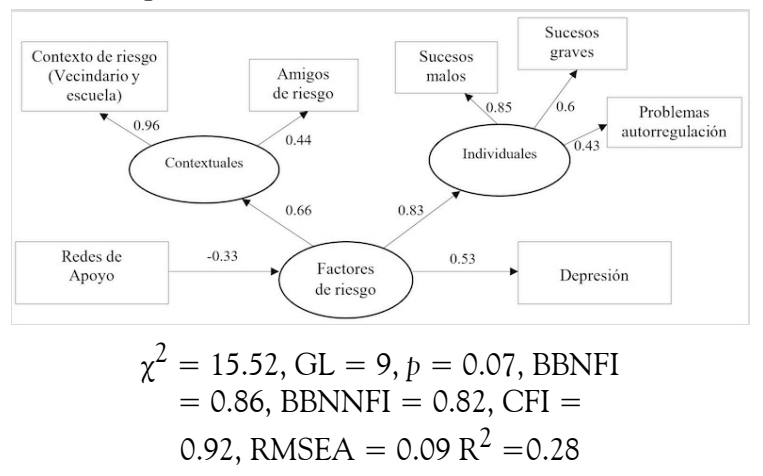

A partir de este resultado, se eliminó la variable latente de tercer orden con menor peso estructural (problemas de autorregulación = 0.43), obteniéndose un segundo modelo descrito en la Figura 3, con indicadores de bondad de ajuste superiores a 0.95 , un valor de chi cuadrado no significativo, cuya razón entre los grados de libertad es menor a $3\left(\chi^{2} / g l=1.12\right)$, RMSEA inferior a 0.08. El modelo explica el $29 \%$ de la depresión como variable dependiente. 
Figura 3

Modelo final con las variables relacionadas a la depresión en adolescentes

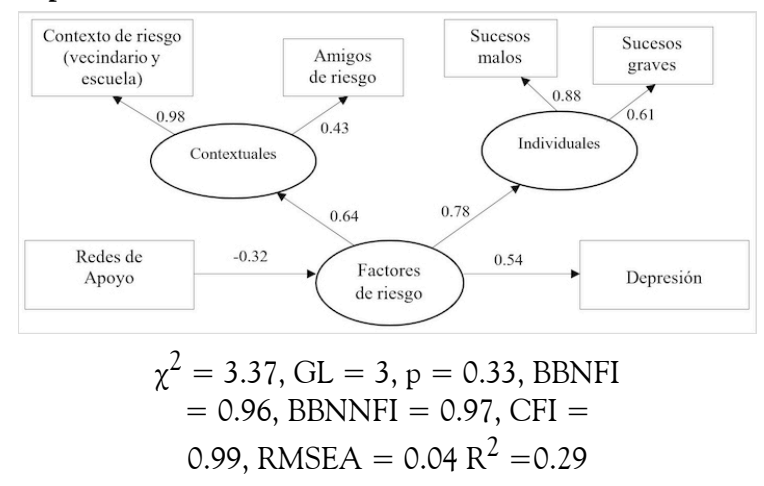

\section{Discusión}

A partir de los datos, se obtuvo un modelo para la depresión que explica el 29 \% de la varianza, lo cual implica que más de una cuarta parte de los indicadores de depresión en la muestra estudiada se explica a partir de este modelo. De tal manera que características individuales y contextuales constituyen factores de riesgo que se relacionan con los indicadores de depresión. Este factor de riesgo para la depresión está conformado por las características del contexto del vecindario y de la escuela, así como por los rasgos negativos de los amigos y, por otro lado, por los sucesos de vida estresantes que reportaron los adolescentes. Este hallazgo apoya los postulados en los cuales la depresión no es el resultado solamente de procesos bioquímicos, sino de un conjunto de elementos psicosociales que interactúan para conformar riesgos que hacen probable el desarrollo de enfermedades mentales (Díaz et al., 2006).

En los factores de riesgo contextuales, las características del vecindario y la escuela tuvieron un mayor impacto como factor de riesgo que las particularidades de los amigos, lo cual coincide con lo reportado por Reynolds y Crea (2015), quienes señalan que los amigos tienen una relación moderada con la depresión. Sin embargo, estos mismos autores reportan que la autorregulación es una variable relacionada, aunque no se ajustó en el modelo planteado del presente estudio. De acuerdo al instrumento utilizado, esta se evaluó en diversas áreas de la vida cotidiana; sin embargo, es posible que quienes presentan síntomas depresivos tengan escasa autorregulación, específicamente para disminuir los pensamientos perturbadores que promueven dichos síntomas (Galicia, Sánchez, \& Robles, 2013). Por lo tanto, no debe descartarse la posible relación entre los problemas de autorregulación y la depresión, sino que se sugiere evaluarla de manera más específica, en relación con la forma de controlar los pensamientos y emociones vinculados a los síntomas depresivos. Las características de riesgo del vecindario y la escuela como factor de riesgo para la depresión resultaron con un alto coeficiente estructural en el modelo, incluso mayor que los sucesos de vida, lo que implica que las condiciones desfavorables de la vivienda, las calles, escuela y la percepción de inseguridad son factores que influyen de manera importante en los sentimientos de tristeza, culpa y pérdida de interés en diversas actividades.

Por otro lado, los sucesos de vida, tanto malos como graves, también conformaron los factores de riesgo relacionados con la depresión, como lo refieren otros estudios con adolescentes mexicanos (Veytia et al., 2012). En resumen, aunque con relaciones causales diferentes, las características negativas percibidas en la escuela, el vecindario y de los amigos influyen en la depresión de los adolescentes, además de los sucesos de vida estresantes, de manera que estos deben evaluarse a la par del contexto en el que suceden (Cutrona et al., 2006), pues ambos elementos tuvieron efectos en los indicadores de depresión.

El modelo resultante apoya el concepto del efecto acumulativo de los factores de riesgo como elemento relacionado con la vulnerabilidad de desarrollar una problemática, donde múltiples factores de riesgo aumentan la probabilidad del resultado indeseado (Elovainio et al., 2015; Evans, Li, \& Whipple, 2013). Finalmente, se observa un efecto negativo entre las redes de apoyo social y los factores de riesgo; por lo tanto, el apoyo proveniente de distintas fuentes (familia, amigos, vecinos, maestros e instituciones) tiene 
un papel protector que mitiga el efecto negativo de dichos factores (Auerbach et al., 2011).

Los hallazgos sugieren la elaboración de programas sociales y políticas públicas que promuevan la seguridad de los vecindarios y la mejora en la infraestructura de los centros escolares, así como a nivel psicológico, la promoción de competencias individuales como las estrategias de afrontamiento para enfrentar los sucesos de vida estresantes que viven los adolescentes en la actualidad, tales como divorcio de los padres, abandono, problemas escolares, entre otros, ya que el uso de estrategias de afrontamiento activas favorece la adaptación ante eventos de vida estresantes y se considera un factor protector que favorece la resiliencia (Restrepo, Vinaccia, \& Quiceno, 2011).También, se sugiere desarrollar programas de intervención dirigidos a la creación de grupos de apoyo dentro de los centros escolares, donde se promuevan estrategias para enfrentar los sucesos de vida de los adolescentes a la vez que se ofrezca un microcontexto protector que mitigue el efecto de los riesgos y refuerce conductas prosociales, a fin de contrarrestar las conductas de riesgo de los amigos con quienes se interactúa, tanto en la escuela como en el vecindario.

Entre de las limitaciones del estudio se encuentra su naturaleza transversal que impide establecer relaciones causales, así como el tipo y tamaño de la muestra que imposibilita la generalización de resultados. Para futuras investigaciones, se sugiere el uso de muestras aleatorias y representativas para traslapar estos resultados a población no vulnerable. Así mismo, se recomienda analizar de manera diferenciada tanto las fuentes de apoyo como el tipo de suceso que tiene mayor relación con la depresión, a fin de determinar qué sucesos y cuáles apoyos son los más significativos para los adolescentes.

\section{Referencias}

American Psychiatric Association. (2013). Diagnostic and statistical manual of mental disorders (5th. ed.). Washington, DC: Autor.
Auerbach, R. P., Bigda-Peyton, J. S., Eberhart, N. K., Webb, C. A., \& Ho, M. H. (2011). Conceptualizing the prospective relationship between social support, stress, and depressive symptoms among adolescents. Journal of Abnormal Child Psychology, 39(4), 475-487. https://doi.org/ 10.1007/s10802-010-9479-x

Barcelata, B. E., Durán, C., \& Lucio, E. (2012). Valoración subjetiva de los sucesos de vida estresantes en dos grupos de adolescentes de zonas marginadas. Salud Mental, 35(6), 513-520. Recuperado de http://www.revistasaludmental.mx/inde x.php/salud_mental/article/view/1509/150 7

Beck, A. T., Steer, R. A., \& Brown, G. K. (1996). Beck Depression Inventory (2nd. ed.) [Inventario de depresión de Beck (2.a ed.)]. San Antonio, TX: Psychological Corporation.

Benjet, C., Borges, G., Medina-Mora, M. E., Fleiz-Bautista, C., \& Zambrano-Ruiz, A. (2004). La depresión con inicio temprano: prevalencia, curso natural y latencia para buscar tratamiento. Salud Pública de México, 46(5), 417-424. https://doi.org/10.1590/SO 036-36342004000500007

Bentler, P. M. (2006). EQS 6 Structural Equations Program Manual. Encino, CA: Mulivariate Software Inc.

Contreras, J. A., Hernández, L., \& Freyre, M. A. (2015). Validez de constructo del Inventario de Depresión de Beck II para adolescentes. Terapia Psicológica, 33(3), 195-203. Recuperado de http://teps.cl/inde x.php/teps/article/view/6/7

Cutrona, C. E., Russell, D. W., \& Brown, P. A. (2005). Neighborhood context, personality, and stressful life events as predictors of depression among African American women. Journal of Abnormal Psychology, 114(1), 3-15. https://doi.org/10.1037/0021843X.114.1.3

Cutrona, C. E., Wallace, G., \& Wesner, K. A. (2006). Neighborhood characteristics and depression: An examination of stress processes. Current Directions in Psychological 
Science, 15(4), 188-192. https://doi.org/10. 1111/j.1467-8721.2006.00433.x

Díaz, J., Torres, W., Urrutia, E., Moreno, R., Font, I., \& Cardona, M. (2006). Factores psicosociales de la depresión. Revista Cubana de Medicina Militar, 35(3), 1-7. Recuperao de http://scielo.sld.cu/scielo .php? script $=$ sci_arttext\&pid $=$ S0138-6557 2006000300009

Diez Roux, A. V., \& Mair, C. (2010). Neigborhoods and health. Annals of the New York Academy of Sciences, 1186, 125-145. https://doi.org/10.1111/j.1749-66 32.2009.05333.x

Dishion, T. J., \& Tipsord, J. M. (2011). Peer contagion in child and adolescent social and emotional development. Annual Review of Psychology, 62, 189-214. https://doi.org/10.1146/annurev. psych.093008.100412

Elovainio, M., Pulkki-Raback, L., Hakulinen, C., Ferrie, J. E., Jokela, M., Hintsanen, M., ... Keltikangas-Järvinen, L. (2015). Childhood and adolescence risk factors and development of depressive symptoms: The 32-year prospective Young Finns followup study. Journal of Epidemiology and Community Health. https://doi.org/10.1136/ jech-2014-205352

Evans, G., Li, D., \& Whipple, S. S. (2013). Cumulative risk and child development. Psychological Bulletin, 139(6), 1342-1396. h ttps://doi.org/10.1037/a0031808

Frías, M., López, A. E., \& Díaz, S. G. (2003). Predictores de la conducta antisocial juvenil: un modelo ecológico. Estudios de Psicología, 8(1), 15-24. https://doi.org/10.15 90/S1413-294X2003000100003

Galicia, I. X., Sánchez, A., \& Robles, F. J. (2013). Self-efficacy in school age adolescents: Its relationship with depression, academic achievement and family relationships. Anales de Psicología, 29(2), 491-500. https:/ /doi.org/10.6018/analesps.29.2.124691

Gaxiola, J. C., González, S., \& Contreras, Z. (2012). Influencia de la resiliencia, metas y contexto social en el rendimiento académico de bachilleres. Revista
Electrónica de Investigación Educativa, 14(1), 165-181. Recuperado de https://redie.uabc $. \mathrm{mx} / \mathrm{redie} / \mathrm{article} / \mathrm{view} / 306$

Gaxiola, J. C., González, S., Domínguez, G. M., \& Gaxiola, E. (2013). Autorregulación, metas y rendimiento académico en bachilleres con disposiciones resilientes y no resilientes. Revista Interamericana de Psicología, 47(1), 71-82. Recuperado de https://journal.sipsych.org/index.php/IJ P/article/viewFile/203/pdf

Gioia, G., Isquith, P., Retzlaff, P. D., \& Espy, K. A. (2002). Confirmatory factor analysis of the Behavioral Rating Inventory of Executive Function [BRIEF] in a clinical sample. Child Neuropsychology, 8, 294-257. https://d oi.org/10.1076/chin.8.4.249.13513

González, L. S., \& Rivera, S. M. (2016). Efectos del apoyo social, resiliencia y competencia social en el bienestar psicológico de adolescentes de sectores vulnerables,con eventos de vida estresantes. En J. C. Gaxiola \& J. Palomar (Eds.), El bienestar psicológico: una mirada desde Latinoamérica (pp. 31-60). México: Editorial Qartuppi.

Goodman, E., Huang, B., Wade, T. J., \& Kahn, R. S. (2003). A multilevel analysis of the relation of socio-economic status to adolescent depressive symptoms: Does school context matter? Journal of Pediatrics, 143, 451-456. https://doi.org/10.1067/S002 2-3476(03)00456-6

Hankin, B. L. (2015). Depression from childhood through adolescence: Risk mechanisms across multiple systems and levels for analysis. Current Opinion in Psychology, 4, 3-12. https://doi.org/10.1016/j.copsyc.2015 .01 .003

Heredia, M. C., Lucio, E. , \& Suárez de la Cruz, L. E. (2011). Depresión y sucesos de vida estresantes en adolescentes. Revista Latinoamericana de Medicina Conductual, 1(2), 49-57. https://doi.org/10.5461/rlmc.v 1.i2.20704

Huston, A. C., \& Bentley, A. C. (2010). Human development in societal context. Annual Review of Psychology, 61, 
411-437. https://doi.org/10.1146/annurev. psych.093008.100442

Kindt, K. C. M., Kleinjan, M., Janssens, J. M. A., \& Scholte, R. H. J. (2015). Cross-lagged associations between adolescents' depressive symptoms and negative cognitive style: The role of negative life events. Journal of Youth and Adolescence, 44, 2141-2153. https://doi.org/ 10.1007/s10964-015-0308-y

La Greca, A. M., \& Harrison, H. M. (2005). Adolescent peer relations, friendships, and romantic relationships: Do they predict social anxiety and depression? Journal of Clinical Child and Adolescent Psychology, 34(1), 49-61. https://doi.org/10.1207/s1537 4424jccp3401_5

Lowe, G. A., Lipps, G., Gibson, R. C., Halliday, S., Morris, A., Clarke, N., \& Wilson, R. N. (2014). Neighbourhood factors and depression among adolescents in four Caribbean countries. PLoS ONE, 9(4), e95538. https://doi.org/10.1371/journal.po ne.0095538

Lucio, E., León, I., Durán, C., Bravo, E., \& Velasco, E. (2001). Los sucesos de vida en dos grupos de adolescentes de diferente nivel socioeconómico. Salud Mental, 24(5), 17-24. Recuperado de http://www.revistasaludmental.mx/inde x.php/salud_mental/article/view/872/870

Lucio, R., Rapp-Paglicci, L., \& Rowe, W. (2011). Developing an additive risk model for predicting academic index: School factors and academic achievement. Journal of Child Adolescence and Social Work, 28(2), 153-173. https://doi.org/10.1007/s10560-0 10-0222-9

Mair, C., Kaplan, G. A., \& EversonRose, S. A. (2012). Are there hopeless neighborhoods? An exploration of environmental associations between individual-level feelings of hopelessness and neighborhood characteristics. Health and Place, 18(2), 434-439. https://doi.org/1 $0.1016 /$ j.healthplace.2011.12.012

Nieva, V. F., \& Sorra, J. (2003). Safety culture assessment: A tool for improving patient safety in healthcare organizations. Quality Safe Health Care, 12 (Suppl. 2), ii17-ii23. ht tps://doi.org/10.1136/qhc.12.suppl2.ii17

Raffaelli, M., Andrade, F., Wiley, A. R., SanchezArmass, O., Edwards, L. L., \& AradillasGarcia, C. (2013). Stress, social support, and depression: A test of the stressbuffering hypothesis in a Mexican sample. Journal Research Adolescence, 23, 283-289. h ttps://doi.org/10.1111/jora.12006

Ramírez, P., \& Hernández, E. (2012). Resiliencia familiar, depresión y ansiedad en adolescentes en situación de pobreza. Revista de Enfermería del Instituto Mexicano del Seguro Social, 20(2), 63-70. Recuperado de http://www.medigraphic.com/pdfs/enfer meriaimss/eim-2012/eim122b.pdf

Restrepo, C., Vinaccia, S., \& Quiceno, M. (2011). Resiliencia y depresión: un estudio exploratorio desde la calidad de vida en la adolescencia. Suma Psicológica, 18(2), 41-48. Recuperado de http://publicaciones.konradlorenz.edu.c o/index.php/sumapsi/article/view/594

Reynolds, A. D., \& Crea, T. M. (2015). Peer influence processes for youth delinquency and depression. Journal of Adolescence, 43, 83-95. https://doi.org/10.1016/j.adolescenc e.2015.05.013

Rosenquist, J. N., Fowler, J. H., \& Christakis, N. A. (2011). Social network determinants of depression. Molecular Psychiatry, 16(3), 273-281. https://doi.org/10.1038/mp.2010. 13

Ruiz, M. A., Pardo, A., \& San Martín, R. (2010). Modelos de ecuaciones estructurales. Papeles del Psicólogo, 31(1), 34-45. Recuperado de http://www.papelesd elpsicologo.es/pdf/1794.pdf

Secretaría de Educación Pública. (2014). Resultado prueba ENLACE 2014. Sonora. Último grado del bachillerato. Recuperado de http://www.enlace.sep.gob.mx/content/ ms/docs/2014/historico/26_EMedia_2014. pdf

Torres, M., \& Ruiz, A. (2013). Habilidades de resiliencia en estudiantes de educación media superior del Estado de México. En J. 
C. Gaxiola \& J. Palomar (Coords.), Estudios de Resiliencia en América Latina (Vol. 2, pp. 53-69). México: Pearson.

Verbeek, T., Bockting, C. L. H., Beijers, C., Meijer, J. L., Van Pampus, M., \& Burger, H. (2015). Low socio-economic position increases the adverse effect of negative life events on anxiety and depression during pregnancy. European Psychiatry, 30 (Suppl. 1), 742. https://doi.org/10.1016/S0924-933 8(15)30588-5

Veytia-López, M., González-Arratia, N. I., Andrade-Palos, P., \& Oudhof, $\mathrm{H}$. (2012). Depresión en adolescentes: el papel de los sucesos vitales estresantes. Salud Mental, 35(1), 37-40. Recuperado de http://www.revistasaludmental.mx/inde x.php/salud_mental/article/view/1453/145 1

Villalobos-Cano, A. (2009). Desarrollo de una medida de resiliencia para establecer el perfil psicosocial de los adolescentes resilientes que permita poner a prueba el modelo explicativo de resiliencia basado en variables psicosociales (Tesis de maestría inédita). Universidad de Costa Rica, San José de Costa Rica. Recuperado de http://www.binasss.sa.cr/bib liotecas/bhp/textos/tesis11.pdf

Wagner, F. A., González-Forteza, C., SánchezGarcía, S., García-Peña, C., \& Gallo, J. J. (2012). Enfocando la depresión como un problema de salud pública en México. Salud Mental, 35(1), 3-11. Recuperado de http://www.revistasaludmental.mx/inde x.php/salud_mental/article/view/1449/144 7

\section{Notas}

* Artículo de investigación. 